couch their requirements in terms which make assumptions as to how the requirements are going to be met. Although, in historical fact, trades union pressures have probably played a greater part in determining crew composition policies than some managements would care to admit, there are two excellent reasons why most of us expect that a sound solution to the long-range navigation problem will exclude the specialist navigator. The first is that we cannot see how the traditional skills of the specialist navigator can have much application in a system of the speed, accuracy and reliability we want. The second is that the arbitrary limits of responsibility associated with the terms pilot, navigator, flight engineer, radio operator are becoming obsolete and appear to impede the design of operation for most efficient utilization of the human crew. Hence, the removal of the specialist navigator is not part of the operational requirement -it is an implied assumption on how the requirement will be met.

Hampton and Mills state that 'the number of aircraft accidents which occur through flying into mountains is an indication of the need for the aids to operate down to ground level'. I do not recall any airliner in recent years flying into a mountain as a result of the deficiency of long-range navigation. The problem is one which can be covered by short-range navigation systems so that coverage to ground level is surely a desirable rather than an essential feature of an L.R. system.

\title{
Reduction to the Prime Vertical Circle
}

\section{from Charles $\mathrm{H}$. Cotter}

'Surprise has sometimes been expressed by mathematicians who have been led by circumstances to look into the subject of nautical astronomy that no method is practised by sailors for reducing a longitude observation to the prime vertical in the same way that an observation for latitude is reduced to the meridian.'

This quotation forms the opening paragraph of the first of three papers $1,2,3$ by Mr. H. B. Goodwin, R.N., written in I 904-6 relating to the problem of reduction to the prime vertical. The following description of Goodwin's work in this connection may not be without interest in view of the recent interesting paper by D. H. Sadler ${ }^{4}$ in which the author describes the investigations into the same problem made by G. F. Cunningham.

The vast difference, to which Mr. Sadler refers, between proposing and translating into practice, a new navigational table, is exemplified in the case of Goodwin's P.V. Tables. All the intermediate steps between proposing and producing are described with utmost clarity in Goodwin's three papers.

The important difference between the problems of 'Reduction to the Meridian' and 'Reduction to the P.V.' is that in the former, after the body has been reduced to the meridian, the problem of finding latitude is merely one of addition or 
subtraction; but in the latter, after the body has been reduced to the P.V., the problem of finding longitude involves solving a right-angled spherical triangle.

When a heavenly body is near meridian passage its change of altitude is almost proportional to the square of its change in hour angle. This principle is employed in many ex-meridian tables. 5 When a heavenly body is near the prime vertical its change of altitude is almost proportional to its change in hour angle. Goodwin was fond of expressing the motion in altitude of an object near the meridian as one of acceleration; and that of an object near the prime vertical as one of uniform velocity. Goodwin demonstrated the second of these motions along the following lines.

If, by means of Taylor's theorem, an expression is obtained for the change in zenith distance $\Delta z$ due to a given change $\Delta h$ in hour angle, latitude $\phi$ and declination $d$ being constant, the first term of the expression includes the coefficient $\Delta z / \Delta h$ which is equal to $\sin Z \cos \phi$ where $Z$ is the azimuth of the observed body. The second term in the expansion includes the coefficient $\Delta^{2} z / \Delta h^{2}$ which is equal to $\cos Z \cos \phi \cos d \cos \theta / \sin z$ where $\theta$ is the parallactic angle (PXZ).

For a body near the prime vertical, $Z$ may be considered to be a right angle, in which case $\sin Z=\mathrm{r}$, and $\cos Z=0$. Thus, for a body near the prime vertical, approximately $\Delta z=\Delta h \cos \phi$, so that $\Delta h \cos \phi$ may be considered to be the velocity at which, in a given latitude $\phi$, a heavenly body is rising or falling when it is near or on the prime vertical circle. After this interesting demonstration Goodwin proceeded as follows.

Consider a projection of the celestial sphere on to the plane of the horizon of an observer in latitude $\phi \mathrm{N}$., whose zenith is projected at $\mathrm{Z}, \mathrm{X}$ is the projection of a celestial body of declination $d \mathrm{~N} .(d<\phi)$, and whose hour angle is $h$, and whose diurnal circle crosses the prime vertical circle at $\mathrm{Y}$.

From PZY

$$
\begin{gathered}
\cos h=\cot \phi \tan d \\
\cos z=\operatorname{cosec} \phi \sin d
\end{gathered}
$$

For small values of $\Delta h, \Delta z$ and $\Delta Z$,

$$
\Delta \mathrm{z}=\Delta h \cos \phi
$$

The change in zenith distance in one minute of time may readily be found from the traverse tables: this being equivalent to ${ }_{15} \cos \phi$ minutes of arc. This change in zenith distance divided into the difference between the observed zenith distance and the zenith distance calculated from (2) gives the time from or to that of passage across the P.V. Goodwin illustrated the process in the following example:

Example. In lat. $47^{\circ} 30^{\prime} \mathrm{N}$. about $5^{\mathrm{h}} \mathrm{r} 0^{\mathrm{m}} \mathrm{p}$.m. when the Sun's declination was $10^{\circ} 37^{\prime} \mathrm{N}$., the observed zenith distance near the P.V. was $73^{\circ} 10^{\prime}$. Required the H.A.

For $h$

$$
\begin{aligned}
\log \cot \phi & =9.96205^{2} \\
\tan d & =9.272876 \\
\cos h & =9.234928 \\
h & =5^{\mathrm{h}} 20^{\mathrm{ml}} 26^{\mathrm{s}}
\end{aligned}
$$

for $z$

$$
\begin{aligned}
\log \operatorname{cosec} \phi & =0.132369 \\
\sin d & =9.265377 \\
\cos z & =9.397746 \\
z & =75^{\circ} 32^{\prime}
\end{aligned}
$$

From traverse tables:

Change of alt. in 1 min. of time $=10^{\circ} 14^{\prime}$ 
Therefore:

$$
X P Y=-\left(\frac{75^{\circ} 32^{\prime}-73^{\circ} 10^{\prime}}{10^{\circ} 14}\right)
$$

(N.B. the negative sign being taken because, from the data, the body had obviously not reached the P.V.)

$$
\begin{aligned}
& h \mathrm{~m} \mathrm{~s} \\
\mathrm{XPY} & =-1400 \\
\mathrm{ZPY} & =052026 \\
\mathrm{ZPX} & =050626
\end{aligned}
$$

Goodwin pointed out that the method adapts itself even more completely to the intercept method. In this case $h$ and $z$ are calculated from (I) and (2), and the rate of change of altitude for the latitude used is found from the traverse tables. This rate is then multiplied by $\Delta h$, and the product applied to $z$ found from (2). This gives the calculated zenith distance which, after comparison with the observed zenith distance, gives the required intercept.

Goodwin's method, outlined above, is limited for use with only those heavenly bodies that cross the P.V. So that all bodies whose declinations are of different denomination from that of the latitude, and those whose declinations are of the same denomination as but greater in magnitude than the latitude, are not available for use with the method.

An object whose declination is the same name as, but is greater in magnitude than, an observer's latitude, reaches its greatest azimuth when its parallactic angle is $90^{\circ}$. At this instant its rate of change of azimuth is zero; and its rate of change of altitude is greatest, being:

$$
\text { I } 5 \cos \phi \sin Z \% \min
$$

Since, at the instant of maximum rate of change of altitude the parallactic angle is $90^{\circ}$, we have, by the sine rule, $\sin Z=\sec \phi \cos d$.

Substituting this value for $\sin Z$ in (3) we have:

$$
\text { Maximum rate of change of altitude }=1.5 \cos d^{\prime} / \mathrm{min} \text {. }
$$

Goodwin treated the problem of finding the hour angle of an object from an observation made at or near the time when the object has maximum azimuth, in a similar way to that for the problem of finding hour angle of an object on or near to the prime vertical. So that, in addition to the problems of 'Reduction to the Meridian' and 'Reduction to the Prime Vertical', there is the third interesting case of what may be termed 'Reduction to the Vertical Circle of greatest Azimuth'.

Goodwin recognized that his treatment of the reduction to the P.V. could be put to profitable use especially in respect of star observations. For this purpose he proposed a table which, as he showed, could be constructed with relatively little labour. 'The labour involved', he stated, 'would be quite insignificant as compared with the gigantic operation with which Mr. Davis (Percy L. H. Davis) and other members of the Nautical Almanac staff have familiarized us'.

Goodwin proposed a table from which the hour angle and zenith distance could be found by inspection against latitude, for each of a number of selected 
stars on the prime vertical. He suggested the use of the following stars :

$\left.\begin{array}{ll}\begin{array}{l}\alpha \text { Andromeda } \\ \alpha \text { Arietis } \\ \text { Aldebaran }\end{array} & \begin{array}{c}\text { Regulus } \\ \beta \text { Leonis } \\ \text { Aetelgeuse } \\ \text { Arcturus } \\ \text { Altair } \\ \text { Markab }\end{array} \\ \begin{array}{l}\text { Rigel } \\ \text { Sirius } \\ \text { Spica }\end{array} & \text { Antares } \\ \text { Formalhaut }\end{array}\right\}$ of north declination

For these stars, and with appropriate latitudes at $\frac{1}{2}^{\circ}$-intervals, some 3000 entries would suffice; this in the space of $6 \mathrm{pps}$. which, Goodwin suggested, might well replace the same number of pages of lunar distances in the Nautical Almanac, which, at the time he wrote, were hardly ever used.

In his second paper, 2 Goodwin described a method of fixing a ship by simultaneous observations of stars, one near the meridian and the other near the P.V. In this paper he suggested that his proposed Reduction to the P.V. Tables should be constructed so that values of $h$ and $z$ are given for $20^{\prime}$-intervals of latitude instead of $30^{\prime}$ as he had originally proposed. He pointed out that the latitude used in the table should never differ by more than $10^{\prime}$ from the estimated latitude. He also suggested that there might usefully be included in this table, columns giving corrections in $h$ and $z$ due to a change of ${ }^{\prime}{ }^{\prime}$ in the declination. Since the declination of a star changes but slowly, the adjustment of the values of $h$ and $z$ could be made at sight; and the table, therefore, would last for several years. Moreover, to obviate the use of the traverse tables, Goodwin suggested a column giving values of change of zenith distance per minute of time.

A fragment of Goodwin's proposed table, printed with his second paper follows :

ELEMENTS OF STARS ON THE PRIME VERTICAL

Arcturus dec. $19^{\circ} 4^{1^{\prime}}$ (1904)

\begin{tabular}{|c|c|c|c|c|c|}
\hline Lat. & H.A. & $\begin{array}{l}\text { corr. per } \\
x^{\prime} \text { of dec. }\end{array}$ & Z.D. & $\begin{array}{l}\text { corr. per } \\
I^{\prime} \text { of dec. }\end{array}$ & $\begin{array}{c}\text { ch. of Z.D. } \\
\text { per min. }\end{array}$ \\
\hline$\circ$, & $\mathrm{h} \mathrm{m} \mathrm{s}$ & $\mathbf{s}$ & - , & ' & , \\
\hline 5420 & $5 \circ 30$ & $3 \cdot 4$ & 6530 & $1 \cdot 3$ & $8 \cdot 75$ \\
\hline 40 & 114 & $3 \cdot 3$ & 37 & $1 \cdot 3$ & .67 \\
\hline 55 & $15^{8}$ & $3 \cdot 3$ & 43 & $1 \cdot 3$ & .60 \\
\hline 20 & 242 & $3 \cdot 2$ & 49 & $1 \cdot 3$ & $\cdot 53$ \\
\hline 40 & 326 & $3 \cdot 2$ & 56 & $1 \cdot 2$ & $\cdot 46$ \\
\hline $5^{6}$ & 49 & $3 \cdot 1$ & 6602 & $1 \cdot 2$ & $\cdot 39$ \\
\hline
\end{tabular}

Goodwin was led to the conclusion that the limits of time from P.V. were wide, stating that from $30-40$ minutes of time on either side of the P.V. the error arising from the approximate nature of the process employed would not, in general, exceed 4 seconds of time.

Goodwin's third paper $^{3}$ contains a summary of his earlier papers on the subject, and includes details of his further investigations into reduction to P.V. 
tables. He had, by this time, drawn up tables, and they were almost ready for publication. The author had evidently given more thought to his previous opinion of the useful range of the tables. He informs us that his earlier opinion-in which he stated that reliable results could be expected for a range of $30-40$ minutes of hour angle on each side of the P.V. - took into account only the first two terms in the series connecting changes of zenith distance and hour angle. Although the second term in this series vanishes when $Z$ is $90^{\circ}$, the third term, which includes $d^{3} z / d h^{3}$, does not vanish. The third term reduces to $-1 / 6 \sin ^{2} 1^{\prime} \sin c \cos ^{2} c(15 t)^{3}$ where $c$ is the co-latitude and $t$ the approximate interval in minutes of time before or after passage of the P.V. Taking this expression into account, a correction in seconds to be applied to the hour angle, as found from the observed zenith distance, may easily be tabulated. With this correction table Goodwin pointed out that the range of the table is practically doubled.

Goodwin's Position Line Star Tables, published by Potter at 5/--, appeared before the close of 1906 . The tables, of about 1 io pages, provide the navigator with the means of fixing his ship by reduction to the meridian and to the prime vertical, without logarithmic computation. It is equally adapted to both the intercept and the 'longitude' methods.

Comparison of the investigations made by Mr. H. B. Goodwin, R.N., and Dr. Cunningham into the very interesting problem of reduction to the $P$.V. reveals the similar lines of attack separated by about half a century in time. The notable difference between the solutions provided is that Dr. Cunningham provides for the azimuth as well as for hour angle and zenith distance. Goodwin, in view of his obvious admiration for Burdwood and his successors, probably thought that Davis' tables were sufficient for the purpose of finding the azimuth.

Goodwin is the author of several works on navigation including Alpho Beta Gamma Navigation Tables (1921) and An Equatorial Azimuth Table (1921), as well as Position Line Star Tables. In addition to these, numerous articles of great interest and value to the student of the history of nautical astronomy appeared under his name during the period c. $1895^{-1930 .}$

\section{REFEREN CES}

1 Goodwin, H. B. (1904). On reduction to the P.V. Naut. Mag.

2 Goodwin, H. B. (1905). On finding position lines by star altitudes. Naut. Mag.

3 Goodwin, H. B. (1906). Prime vertical reduction tables. Naut. Mag.

4 Sadler, D. H. Astrofix and rectofix tables. This Journal, 17, 17.

5 Cotter, C. H. An historical review of the ex-meridian problem. This Journal, $17,72$.

\section{Astrofix and Rectofix Tables}

\section{Mr. D. H. Sadler writes:}

(I) Through the above contribution by Captain Charles H. Cotter, my attention has been drawn to the fact that the essential principle of the 'Rectofix Tables' is not only quite old but has been incorporated in a set of published tables -the Position Line Star Tables (for fixing ship's position by reduction to meridian and prime vertical without logarithmic calculation) by H. B. Goodwin, R.N., J. D. Potter, London, r 906. 